\title{
ESTUDO DOS COMPOSTOS NITROGENADOS E OXIGENADOS OBTIDOS A PARTIR DA PIRÓLISE TÉRMICA DO LODO DE ESGOTO ANAERÓBIO COLETADO NA ETE-MANGUEIRA
}

\author{
S. T. S. VERAS ${ }^{1}$, I. A. T. A. RIBEIRO ${ }^{2}$, J. C. NERIS ${ }^{1}$ e J. G. A. PACHECO FILHO ${ }^{1}$ \\ ${ }^{1}$ Universidade Federal de Pernambuco, Departamento de Engenharia Química \\ ${ }^{2}$ Universidade Federal de Pernambuco, Centro de Ciências Biológicas \\ E-mail para contato: shyrlane_veras@hotmail.com
}

\begin{abstract}
RESUMO - Como o lodo de esgoto é rico em matéria orgânica, ele tem sido reportado como fonte para produção de calor, biocombustíveis e matéria-prima. No entanto, sua conversão em biocombustíveis é limitada devido à quantidade de oxigênio e nitrogênio nos compostos produzidos. O objetivo deste trabalho foi detalhar entre os produtos de pirólise, os compostos oxigenados e nitrogenados obtidos com a micropirólise rápida a $600^{\circ} \mathrm{C}$ de duas amostras de lodo de esgoto anaeróbio (L1 e L2) com diferentes características em relação à composição. A amostra L1 com menor quantidade de matéria orgânica, porém com menor percentual de elementos inorgânicos interferentes no processo de pirólise, apresentou menor percentual de compostos oxigenados, exceto nitrogenados como: nitrilas $\left(\mathrm{C}_{15}-\mathrm{C}_{19}\right)$, aminas aromáticas e aromáticos heterocíclicos (indol e derivados) em relação à amostra L2. Também foi observada uma menor produção de ácidos, resultando em uma maior quantidade de hidrocarbonetos para a amostra L1. A partir deste estudo, observou-se a variabilidade dos produtos pirolíticos obtidos diante de diferenças na composição das amostras.
\end{abstract}

\section{INTRODUÇÃO}

A pirólise é considerada uma alternativa promissora para converter resíduos orgânicos como o lodo de esgoto em produtos gasosos, líquidos e sólidos com valor agregado. No entanto, o bio-óleo produzido tem apresentado quantidade elevadas de compostos nitrogenados, que geralmente são convertidos em $\mathrm{NO}_{\mathrm{x}}$ e $\mathrm{N}_{2} \mathrm{O}$ quando o bio-óleo é empregado como combustível. Além destes, espécies contendo oxigênio também afetam a qualidade e desempenho do bio-óleo. Desta forma, a identificação e remoção destes compostos tornam-se necessários (CAO et al., 2010).

Estudos têm reportado a presença de compostos orgânicos nitrogenados como: piridina, pirrol, indol, pirazinas, aminas, amidas e nitrilas, sendo os compostos heterocíclicos e as nitrilas, obtidos em maior quantidade (CAO et al., 2010; ZHANG et al., 2011). Entre os compostos orgânicos oxigenados, podem ser encontradas espécies pertencentes a funções orgânicas como: ácidos carboxílicos, alcoóis, cetonas, aldeídos, ésteres e fenóis. Muitos estudos sugerem a aplicação de catalisadores ou reações como desoxigenação e hidrodenitrogenação para promover a remoção de oxigênio e nitrogênio do bio-óleo resultante da pirólise (BU et al., 2012; IZHAR et al., 2012). 


\section{9 a 22 de outubro de 2014 \\ Florianópolis/SC}

A identificação dos produtos pirolíticos geralmente é feita através da cromatografia gasosa/ espectroscopia de massa (GC/MS). No entanto, a caracterização destas espécies é dificultada diante da complexidade na estrutura e composição dos componentes orgânicos, principalmente os que são produzidos em menor quantidade. Então, alguns autores utilizam técnicas complementares como análises de infravermelho para auxiliar a identificação dos compostos (FONTS et al., 2009; POKORNA et al., 2009). No presente trabalho, foi dado destaque aos compostos orgânicos nitrogenados e oxigenados obtidos através da micropirólise rápida de duas amostras lodo de esgoto anaeróbio, coletadas na mesma estação de tratamento (ETE-Mangueira) e identificadas através destas técnicas citadas.

\section{METODOLOGIA}

\subsection{Caracterização das Amostras}

As amostras de lodo foram coletadas no leito de secagem da ETE Mangueira, que utiliza o processo de tratamento anaeróbio através de reatores tipo UASB. Depois disso, as mesmas passaram por um processo manual para separação de resíduos grosseiros, moagem, peneiramento (em malha de 120 mesh) e homogeneização. A determinação do teor de umidade, material volátil e fixo foi feita tomando como base a ASTM D2216-98 (2005). Para a identificação de metais e componentes inorgânicos, foram realizadas análises de EDX com espectrômetro de raio-X da Shimadzu, modelo EDX-700. Os percentuais de carbono, hidrogênio, nitrogênio e enxofre da amostra foram determinados através da análise elementar (CE Instruments - Modelo EA110). Por último, foram feitas análises de infravermelho com Transformada de Fourier (FTIR) em um equipamento da marca Bruker (modelo tensor 27), obtendo espectros na faixa de $4000-500 \mathrm{~cm}^{-1}$. Estas análises foram feitas com 150 scans e resolução de $4 \mathrm{~cm}^{-1}$ para determinar os principais grupos funcionais presentes nas amostras.

\subsection{Condições Experimentais das Pirólises Rápidas}

O processo de pirólise foi analisado através de testes com micropirólise rápida do lodo de esgoto, realizada em um micropirolisador CDS Pyroprobe 5200, acoplado a um cromatógrafo a gás com espectrômetro de massa Shimadzu GC/MS QP 2010 Plus, usando como gás de arraste o hélio, coluna DB-5MS com espessura de $0,25 \mu \mathrm{m}, 30 \mathrm{~m}$ de comprimento e diâmetro de $0,25 \mathrm{~mm}$; a qual foi submetida a uma temperatura inicial de $40^{\circ} \mathrm{C}$ por 5 minutos, seguida de uma rampa de aquecimento sob uma taxa de $5^{\circ} \mathrm{C} \min ^{-1}$ até atingir $300^{\circ} \mathrm{C}$, na qual permaneceu por 30 minutos. A fonte de íons foi mantida a $230^{\circ} \mathrm{C}$ e a interface a $250^{\circ} \mathrm{C}$, sendo a análise realizada no modo scan adquirindo massas no intervalo de $25-533 \mathrm{~m} / \mathrm{z}$. A pirólise foi realizada a $600^{\circ} \mathrm{C}$, numa taxa de aquecimento teórica de $10^{\circ} \mathrm{C} . \mathrm{ms}^{-1}$ e um tempo total de aquecimento de 40 segundos. A interface do pirolisador foi aquecida a $110^{\circ} \mathrm{C}$ por 1 minuto, para secar a biomassa, aumentando-se para $325^{\circ} \mathrm{C}$ por 4 minutos. A linha de transferência foi mantida a $325^{\circ} \mathrm{C}$. Os produtos de reação foram identificados com ajuda da biblioteca de compostos NIST de espectros de massa. As análises foram feitas em duplicata e considerados apenas os 70 picos principais com área percentual igual ou superior a $0,5 \%$. Os experimentos foram feitos em duplicata e para cada produto identificado, foi utilizado o valor médio referente à área percentual dos picos, conforme realizado no trabalho de Lu et al. (2011). 


\section{RESULTADOS E DISCUSSÃO}

\subsection{Caracterização das Amostras de Lodo de Esgoto Anaeróbio}

Os resultados das análises iniciais para caracterização das amostras como: umidade, matéria orgânica (volátil), fixa e percentual de carbono $(\mathrm{C})$, hidrogênio $(\mathrm{H})$ nitrogênio $(\mathrm{N})$ e enxofre $(\mathrm{S})$ estão apresentados na Tabela 1. A partir dela é possível observar que a amostra L2 apresentou maiores percentuais de matéria orgânica $(42,6 \%)$ e consequentemente, maior percentual de carbono em relação à amostra L1. No entanto, a diferença entre estes valores não é grande. Diante disto, as amostras podem gerar diferentes produtos de pirólise devido à interferência de outros fatores durante o processo.

Tabela 1 - Parâmetros de caracterização das amostras de lodo (L1 e L2)

\begin{tabular}{lrr}
\hline Parâmetro & L1 & L2 \\
\hline Umidade (\%) & 6,5 & 4,3 \\
Material em base seca (\%) & 93,5 & 95,7 \\
Material Volátil (\%) & 35,0 & 42,6 \\
Material Fixo (\%) & 58,5 & 53,1 \\
C (\%) & 18,8 & 20,9 \\
H (\%) & 3,2 & 3,3 \\
N (\%) & 2,1 & 2,5 \\
S (\%) & 1,8 & 2,5 \\
\hline
\end{tabular}

Segundo Akhtar e Amin (2012), Park et al. (2010) e Shao et al. (2010) elementos como Ca, K, $\mathrm{Fe}, \mathrm{Zn}$ e Ti nas amostras a serem pirolisadas poderiam proporcionar a aceleração das reações de desidratação e carbonização, além do aumento no tempo de pirólise para decompor a matéria orgânica. Esses efeitos geralmente resultam na redução da produção de bio-óleo, fração de interesse quando se deseja a aplicação da pirólise para obtenção de biocombustíveis. Devido a isto, foram feitas análises de EDX para identificar os principais componentes inorgânicos presentes nas amostras de lodo antes de pirolisá-las. Os resultados estão apresentados na Tabela 2.

Tabela 2 - Percentual médio de componentes inorgânicos nas amostras de lodo.

\begin{tabular}{ccccccccccccc}
\hline \multirow{2}{*}{ Amostra } & \multicolumn{10}{c}{ Quantidade (\%) } \\
\cline { 2 - 13 } & $\mathrm{Fe}$ & $\mathrm{Si}$ & $\mathrm{Ca}$ & $\mathrm{S}$ & $\mathrm{Zn}$ & $\mathrm{K}$ & $\mathrm{Ti}$ & $\mathrm{Cu}$ & $\mathrm{Sr}$ & $\mathrm{Mn}$ & $\mathrm{P}$ & $\mathrm{Zr}$ \\
\hline $\mathbf{L 1}$ & 26,6 & 43,1 & 12,4 & 5,2 & 1,3 & 2,8 & 1,5 & 0,3 & 0,3 & 0,2 & 5,6 & N.I. \\
L2 & 29,1 & 31,4 & 17,9 & 9,7 & 1,7 & 2,6 & 1,6 & 0,4 & 0,3 & 0,4 & 4,4 & 0,4 \\
\hline
\end{tabular}

A partir destes resultados, foi possível identificar maiores percentuais de $\mathrm{Fe}, \mathrm{Ca}, \mathrm{Zn}$ e Ti para a amostra L2, podendo fornecer menor rendimento, principalmente em hidrocarbonetos gerados, mesmo apresentando maior quantidade de matéria orgânica em relação à L1. Ainda como parte da caracterização das amostras, foram feitas análises de infravermelho com Transformada de Fourier (FTIR) para identificar os principais grupos funcionais presentes nas mesmas. Como o lodo de esgoto apresenta uma constituição complexa, várias bandas foram observadas conforme mostra a Figura 1. 


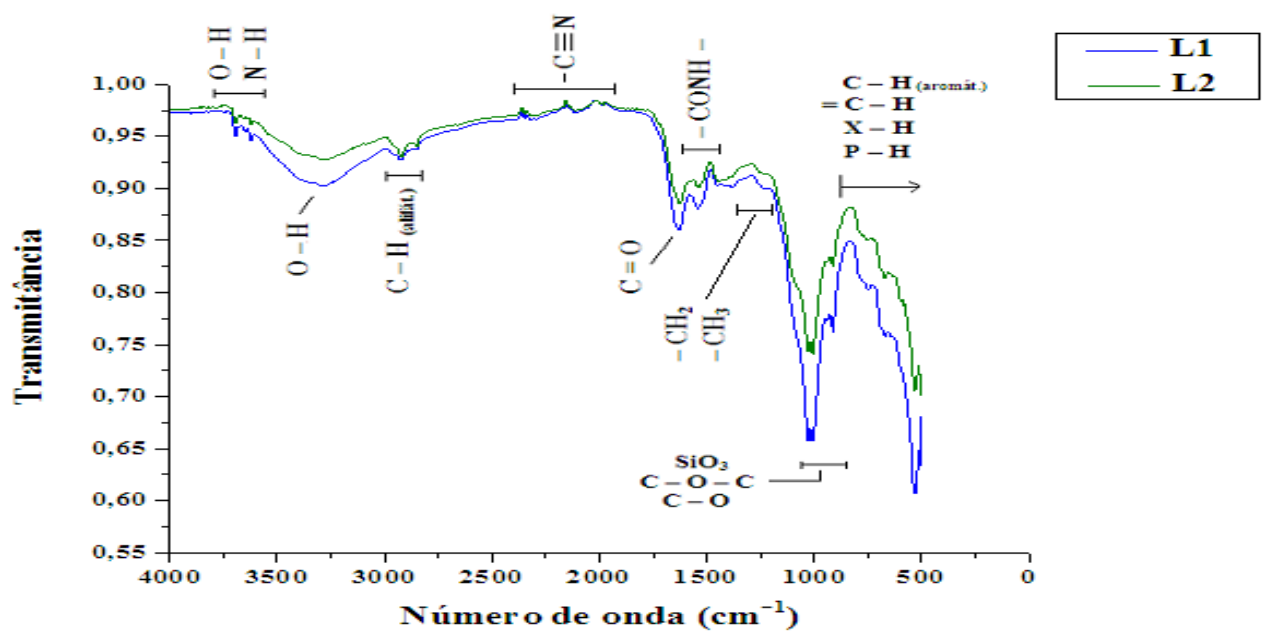

Figura 1 - Espectros de infravermelho das amostras de lodo L1 e L2.

Pela Figura 1, observaram-se bandas atribuídas às ligações $\mathrm{O}-\mathrm{H}$ e N-H (3800-3000 cm $\left.\mathrm{cm}^{-1}\right)$, indicando a possível presença de água, aminas, alcoóis, fenóis, ácidos carboxílicos e amidas primárias. As ligações $\mathrm{C}-\mathrm{H}$ identificadas $\left(3000-2800 \mathrm{~cm}^{-1}\right)$, geralmente indicam a existência de cadeias alifáticas. As bandas observadas entre $2300-2000 \mathrm{~cm}^{-1}$, foram atribuídas às ligações $\mathrm{C} \equiv \mathrm{N} \mathrm{e}-$ $\mathrm{N}=\mathrm{C}$, geralmente formadas pela desidratação de ligações peptídicas. Vibrações próximas a $1700 \mathrm{~cm}^{-1}$ sugerem a existência de grupos $\mathrm{C}=\mathrm{O}$, principalmente devido à presença de compostos como ácidos carboxílicos. Grupos -CONH-, característicos das amidas, foram observados entre $1650-1550 \mathrm{~cm}^{-1} \mathrm{e}$ podem ser formados pela desnaturação de proteínas. Alguns autores, como Fonts et al. (2009), afirmaram que entre 1600-1500 $\mathrm{cm}^{-1}$, também é possível encontrar grupos que indicam a presença de alcenos e aromáticos. A existência de grupos $-\mathrm{CH}_{2}$ e $-\mathrm{CH}_{3}$, característicos dos alcanos, foram sugeridas entre 1500 e $1300 \mathrm{~cm}^{-1}$. Esses grupos são encontrados na celulose, que por sua vez, fazem parte da composição do lodo de esgoto. As vibrações entre $1150-1000 \mathrm{~cm}^{-1}$ podem estar associados a polissacarídeos, $1080 \mathrm{~cm}^{-1}$ a silicatos e $1064 \mathrm{~cm}^{-1}$ às ligações $\mathrm{C}-\mathrm{O}$ e $\mathrm{C}-\mathrm{O}-\mathrm{C}$. Geralmente vibrações observadas entre 1120-1045 $\mathrm{cm}^{-1}$, também podem ser atribuídos a grupos C-NH-C (isonitrilas). Já as bandas observadas entre $900-500 \mathrm{~cm}^{-1}$, foram atribuídas a existência de grupos $\mathrm{C}-\mathrm{H}_{\text {Aromáticos, }}$, sendo o hidrogênio ligado a grupos aromáticos. Essa faixa é citada pela literatura como menos representativa, porém segundo Fonts et al. (2009), ela pode indicar a presença de estruturas cíclicas alifáticas, aromáticas e compostos contendo halogênios e fósforo em sua estrutura. Em geral, as amostras apresentaram os mesmos grupos funcionais, diferindo apenas em relação à transmitância dos espectros, sendo a amostra L1 com menores valores para a mesma.

\subsection{Produtos Nitrogenados e Oxigenados}

Como pode ser visto na Figura 2, a amostra L1 com menor quantidade de matéria orgânica, forneceu maior percentual de hidrocarbonetos e $\mathrm{CO}_{2}$ e uma menor quantidade de ácidos, sugerindo a ocorrência de reações de descarboxilação como ocorre na pirólise de triglicerídeos (KUBICKOVA; KUBICKA, 2010). Além disto, conforme citado (Tabela 2), pode ter ocorrido influências de elementos inorgânicos, já que a amostra L2 (com maior quantidade de matéria orgânica) gerou menor 
percentual de hidrocarbonetos. Os demais produtos identificados comprovaram a presença de compostos que as análises de FTIR sugeriram.

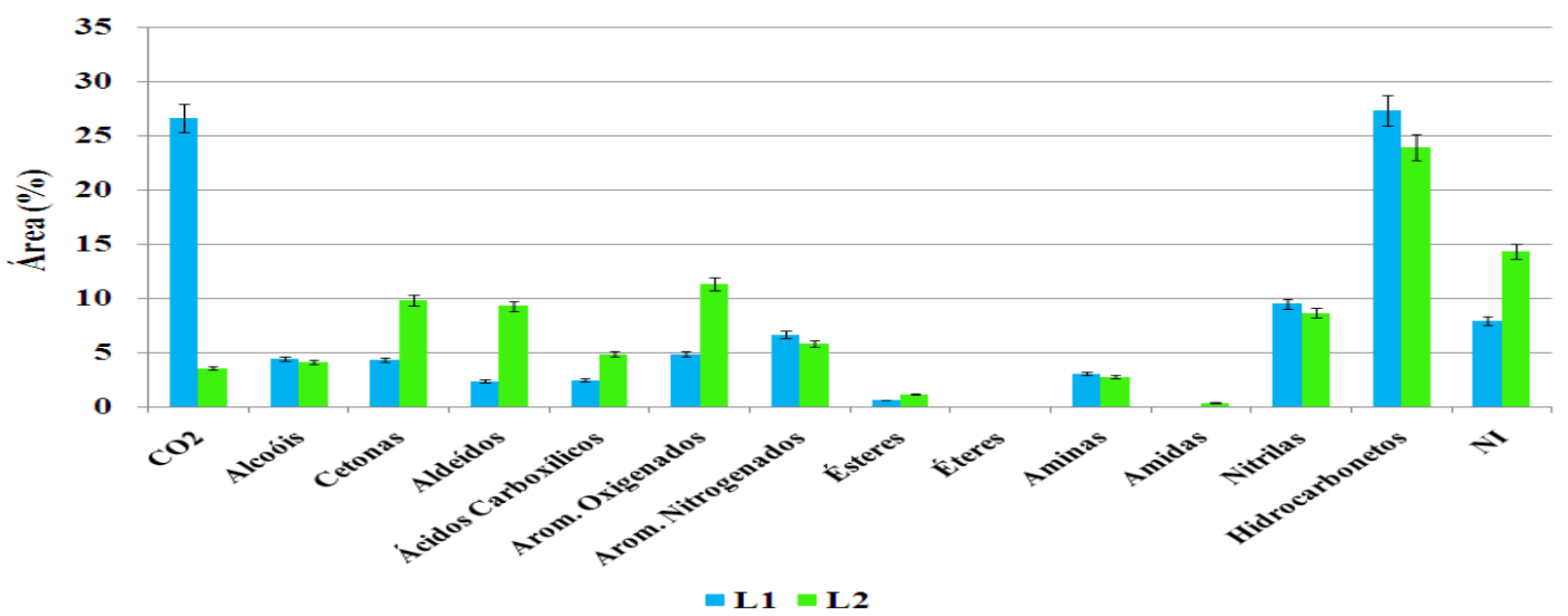

Figura 2 - Distribuição geral dos produtos pirolíticos das amostras L1 e L2 a $600^{\circ} \mathrm{C}$.

Sendo NI = Não identificado.

Para detalhar os compostos orgânicos contendo nitrogênio e oxigênio em sua composição, foram construídos gráficos para mostrar os principais componentes, conforme mostram as Figuras 3 e 4.

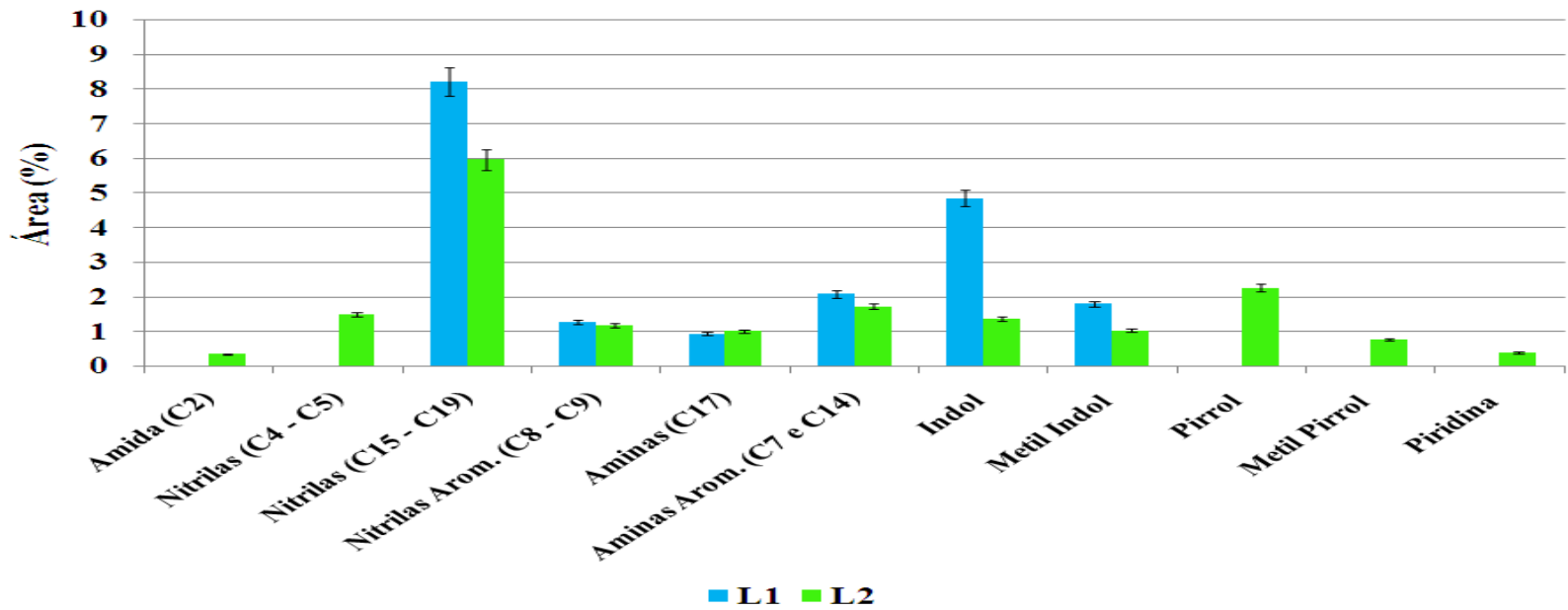

Figura 3 - Compostos nitrogenados obtidos com a micropirólise das amostras de lodo L1 e L2.

A amostra L2, forneceu uma maior variedade de compostos nitrogenados como: pirrol, metil pirrol, piridina, amida (acetamida) e nitrilas com baixo peso molecular em relação à L1. Esta última, por sua vez, apresentou maiores percentuais para nitrilas com maior peso molecular, indol, metil indol e aminas aromáticas (Figura 3). Estes produtos são derivados da proteína que é um dos principais constituintes do lodo de esgoto. Segundo Zhang et al. (2011), muitas vezes o hidrogênio é responsável pela quebra das ligações peptídicas durante o processo de pirólise. 
O pirrol é citado como derivado do aminoácido prolina. Enquanto o indol e metil indol são referenciados como resultantes da pirólise do triptofano e geralmente são os nitrogenados obtidos em maior quantidade na fração líquida da pirólise de lodo de esgoto. Já a acetamida provavelmente é produto da pirólise de quitina, proteínas termolábeis e amino-açúcares (CAO et al., 2010; FONTS et al., 2009). A diferença entre os produtos obtidos para as amostras pode estar relacionada à variação na composição das amostras (L1 e L2), principalmente proteínas.

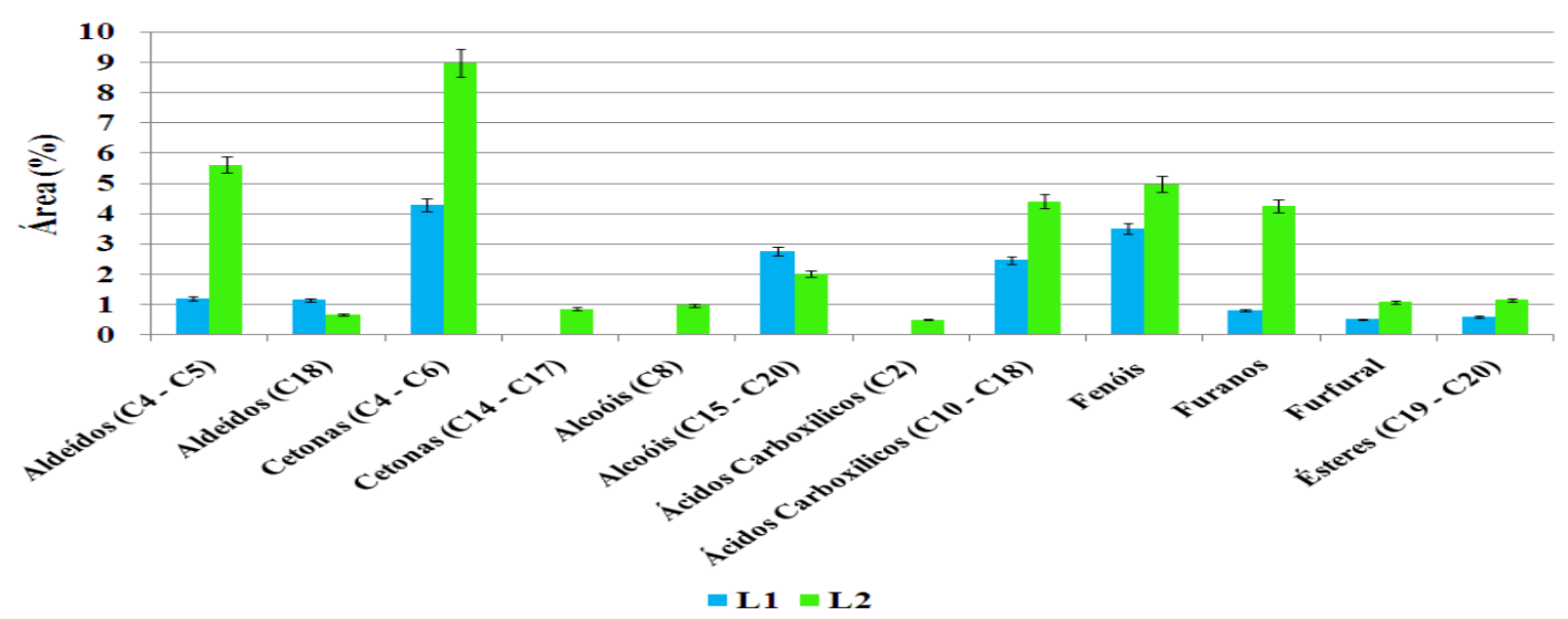

Figura 4 - Compostos oxigenados obtidos com a micropirólise das amostras de lodo L1 e L2.

No caso da amostra L1, a redução na quantidade de compostos orgânicos contendo oxigênio pode estar associada com a ocorrência de reações de desoxigenação, contribuindo também com uma maior produção de $\mathrm{CO}_{2}$, conforme verificado (Figuras 2 e 4). Pela Figura 4, é possível observar que a amostra de lodo anaeróbio L2, também produziu uma maior variedade de compostos oxigenados. A única exceção foi com relação ao octadecenal (aldeído).

Autores como $\mathrm{Bu}$ et al. (2012) e Lu et al. (2011) associam a obtenção de compostos oxigenados, principalmente fenóis, à pirólise da celulose que também faz parte da constituição do lodo de esgoto. O processo de pirólise da parte celulósica do lodo pode fornecer produtos como: furanos, fenóis, ésteres, cetonas e açúcares anidro. Sendo assim, a amostra de lodo L2 pode conter uma fração de material celulósico maior em relação à L1. A amostra L2 também formou maior quantidade em compostos com baixo peso molecular, o que pode estar associado à presença de sais minerais. Segundo Patwardhan et al. (2010), sais minerais, principalmente os que contêm Ca, Na, K e $\mathrm{Mg}$, podem favorecer a formação de produtos pirolíticos com baixo peso molecular.

Os mecanismos das reações que ocorrem durante o processo de pirólise ainda vêm sendo estudados, pois devido à grande quantidade de compostos, apresentar um mecanismo exato é algo bastante complexo. O que alguns estudos mostram, são basicamente sugestões ou possíveis reações que podem ocorrer durante o processo. Algumas pesquisas como as desenvolvidas por Sanchéz et al. (2009) e Zhang et al. (2011), têm sugerido que os mecanismos de reação de Diels-Alder e reações secundárias entre compostos aromáticos oxigenados como fenóis são responsáveis pela formação de hidrocarbonetos poliaromáticos (PAHs) na fração líquida obtida durante a pirólise (reação 1 - 3), o 
que pode justificar a menor quantidade de fenóis e maior quantidade de hidrocarbonetos formados através da amostra L1.

- Desidrogenação de alcanos a alcenos/dienos:

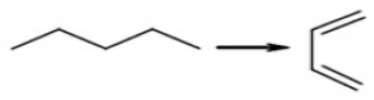

- Formação de compostos cíclicos:

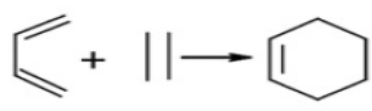

- Exemplo para a formação de PAHs:

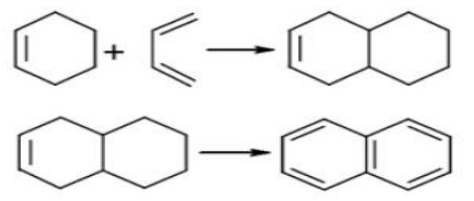

\section{CONCLUSÃO}

O presente trabalho mostrou que a pirólise de lodo pode gerar diferentes produtos mesmo sendo empregada em amostras coletadas na mesma estação de tratamento. Neste estudo, a amostra L1 com menor quantidade de matéria orgânica e menor quantidade de elementos inorgânicos gerou maior percentual de hidrocarbonetos, o que pode estar associado à reação de descarboxilação, visto que foram obtidos menores percentuais para os ácidos carboxílicos e elevada quantidade de $\mathrm{CO}_{2}$. Além disto, a menor obtenção de fenóis para esta amostra também pode ter contribuído para uma maior quantidade de hidrocarbonetos. A amostra L2, por sua vez, apresentou maior variedade de compostos nitrogenados e oxigenados em relação à $\mathrm{L} 1$, principalmente com baixo peso molecular como: amidas $\left(\mathrm{C}_{2}\right)$, nitrilas $\left(\mathrm{C}_{4}-\mathrm{C}_{5}\right)$, aldeídos $\left(\mathrm{C}_{4}-\mathrm{C}_{5}\right)$, cetonas $\left(\mathrm{C}_{4}-\mathrm{C}_{6}\right)$ e alcoóis $\left(\mathrm{C}_{8}\right)$. De modo geral, foi possível observar a maior formação de produtos pirolíticos comuns na pirólise de celulose (compostos oxigenados) durante a pirólise da amostra de lodo L2. Enquanto a amostra L1, apresentou maior percentual de nitrogenados, principalmente nitrilas $\left(\mathrm{C}_{15}-\mathrm{C}_{19}\right)$, nitrilas aromáticas $\left(\mathrm{C}_{8}\right.$ e $\left.\mathrm{C}_{9}\right)$ e aromáticos heterocíclicos, produtos que normalmente são obtidos através da pirólise de proteínas e aminoácidos. Estes resultados, comprovaram que a variação na composição da biomassa é um importante fator interferente no processo de pirólise, podendo provocar alterações significantes nos produtos mesmo com diferença percentual pequena dos parâmetros de caracterização da biomassa (umidade, sólidos, etc.).

\section{REFERÊNCIAS}

AKHTAR, J.; AMIN, N. S. A review on operating parameters for optimum liquid oil yield in biomass pyrolysis. Renew. Sust. Energ. Rev., v. 16, p. 5101-5109, 2012.

ASTM D2216-98, Standard Test Method for Laboratory Determination of Water (Moisture) Content of Soil and Rock by Mass, ASTM, USA, 2005. 
BU, Q.; LEI, H.; REN, S.; WANG, L.; ZHANG, Q.; TANG, J.; RUAN, R. Production of phenols and biofuels by catalytic microwave pyrolysis of lignocellulosic biomass. Bioresource Technol., v. 108, p. 274-279, 2012.

CAO, J. P.; ZHAO, X. Y.; MORISHITA, K.; WEI, X. Y.; TAKARADA, T. Fractionation and identification of organic nitrogen species from bio-oil produced by fast pyrolysis of sewage sludge. Bioresource Technol., v. 101, p.7648-7652, 2010.

FONTS, I.; AZUARA, M.; GEA, G.; MURILLO, M. B. Study of the pyrolysis liquids obtained from different sewage sludge. J. Anal. Appl. Pyrol., v. 85, p.184-191, 2009.

IZHAR, S.; UEHARA, S.; YOSHIDA, N.; YAMAMOTO, Y.; MORIOKA, T.; NAGAI, M. Hydrodenitrogenation of fast pyrolysis bio-oil derived from sewage sludge on $\mathrm{NiMo} / \mathrm{Al}_{2} \mathrm{O}_{3}$ sulfide catalyst. Fuel Process. Technol., v. 101, p. 10-15, 2012.

KUBICKOVA, I.; KUBICKA, D. Utilization of triglycerides and related feedstocks for production of clean hydrocarbon fuels and petrochemicals: A review. Waste Biomass Valorization, v. 1, p. 293-308, 2010.

LU, Q.; YANG, X. C.; DONG, C. Q.; ZHANG, Z. F.; ZHANG, X. M.; ZHU, X. F. Influence of pyrolysis temperature and time on the cellulose fast pyrolysis products: Analytical Py-GC/MS study. J. Anal. Appl. Pyrol., v. 92, p. 430-438, 2011.

PARK, H. J.; HEO, H. S.; PARK, Y. K.; YIM, J. H.; JEON, J. K.; PARK, J.; RYU, C.; KIM, S. S. Clean bio-oil production from fast pyrolysis of sewage sludge: Effects of reaction conditions and metal oxide catalysts. Bioresource Technol., v. 101, p. S83-S85, 2010.

PATWARDHAN, P. R.; SATRIO, J. A.; BROWN, R. C.; SHANKS, B. H. Influence of inorganic salts on the primary pyrolysis products of cellulose. Bioresource Technol., v. 101, p. 4646-4655, 2010.

POKORNA, E.; POSTELMANS, N.; JENICEK, P.; SCHREURS, S.; CARLEER, R.; YPERMAN, J. Study of bio-oils and solids from flash pyrolysis of sewage sludges. Fuel, v. 88, p. 1344-1350, 2009.

SÁNCHEZ, M. E.; MENÉNDEZ, J. A.; DOMÍNGUEZ, A.; PÍS, J. J.; MARTÍNEZ, O.; CALVO, L. F; BERNARD, P. L. Effect of pyrolysis temperature on the composition of the oils obtained from sewage sludge. Biomass Bioenerg., v. 33, p. 933-940, 2009.

SHAO, J.; YAN, R.; CHEN, H.; YANG, H.; LEE, D. H. Catalytic effect of metal oxides on pyrolysis of sewage sludge. Fuel Process. Technol., v. 91, p. 1113-1118, 2010.

ZHANG, B.; XIONG, S.; XIAO, B.; YU, D.; JIA, X. Mechanism of wet sewage sludge pyrolysis in a tubular furnace. Int. J. Hydrogen Energ., v. 36, p. 355-363, 2011. 